\title{
Primary non-Hodgkin lymphoma of the right femur and subsequent metastasis to the left femur: A case report and literature review
}

\author{
JING-YU HU ${ }^{1 *}$, DAN YU ${ }^{1,2^{*}}$ and YAO-HUI WU ${ }^{1}$ \\ ${ }^{1}$ Department of Hematology, Union Hospital, Tongji Medical College, Huazhong University of Science and Technology, \\ Wuhan, Hubei 430022; ${ }^{2}$ Department of Hematology, Wuhan First Hospital, Wuhan, Hubei 430030, P.R. China
}

Received May 11, 2016; Accepted June 29, 2017

DOI: $10.3892 / \mathrm{ol} .2018 .7895$

\begin{abstract}
Non-Hodgkin lymphoma of the bone is rare and typically causes an extensive bone lesion. The present study describes a case of diffuse large B-cell primary non-Hodgkin lymphoma of the bone, which occurred in the right femur, and was initially treated with surgery and chemotherapy. Following a 7-year period of complete remission, a new, similar lesion was identified in the left femur. With both lesions, there was no accompanying destruction of any other bones or organ involvement. Metastasis of PLB to the contralateral side is extremely rare and, to the best of our knowledge, this is the first report of this particular presentation in China or worldwide. We hypothesized that the present situation arose due to mechanisms involving the tumor microenvironment, circulating tumor cells, lymphocyte homing and self-seeding. The present report describes the case in detail, and discusses the possible underlying mechanisms and their potential contribution to the treatment of non-Hodgkin lymphoma, as well as the prevention of metastasis and recurrence, which may be of considerable clinical significance.
\end{abstract}

\section{Introduction}

The majority of cases of non-Hodgkin lymphoma of the bone are classified as diffuse large B-cell lymphoma (DLBCL), and typically derive from the lymph nodes and lymphoid tissues. DLBCL is prone to early distant metastasis, and has an aggressive clinical course, which may include invasion of

Correspondence to: Dr Yao-Hui Wu, Department of Hematology, Union Hospital, Tongji Medical College, Huazhong University of Science and Technology, 1277 Jiefang Avenue, Wuhan, Hubei 430022, P.R. China

E-mail:wyh11035@sina.com

${ }^{*}$ Contributed equally

Key words: lymphocyte homing, self-seeding, primary non-Hodgkin's lymphoma of bone, tumor microenvironment the skeletal, nervous, digestive or respiratory systems. Primary non-Hodgkin lymphoma of the bone (PLB) is an extremely rare disease that accounts for $7 \%$ of all malignant bone tumors, and $4-5 \%$ of all extranodal non-Hodgkin lymphoma $(1,2)$. In addition, PLB accounts for $2 \%$ of all lymphomas in adults and $<1 \%$ of all malignant lymphomas (2-4). The incidence of PLB peaks in patients of $\sim 55$ years of age, and this type of lymphoma exhibits a male preponderance, with a male-to-female ratio of 2:1 (2). The principal areas of destruction in cases of PLB are the tubular bones of the limbs and the axial skeleton; destruction may be observed in the femur, tibia, humerus, and vertebral bodies, and also the shoulder blades, pelvis, ribs and sternum (2,5). Beal et al (6) reported that the femur was the most common site of involvement, accounting for $27 \%$ of 82 cases in a retrospective study of patients with PLB. Additional common sites of involvement included the pelvis (15\%), tibia (13\%), humerus (12\%) and spine (9\%), while other sites accounted for $6 \%$ of cases (6). Wu et al (7) also suggested that the femur was the most common site of unifocal PLB, whereas the spinal bones were more commonly involved in cases of multifocal PLB. Patients with PLB typically exhibit a good prognosis and present with mild clinical symptoms, including localized bone pain and, less commonly, systemic symptoms, such as fever, emaciation and night sweats (5). Relapse of PLB is characterized by spread of the disease to the central nervous system, but this is rare (2).

The incidence of PLB is extremely low, and the metastasis of PLB from one long bone to the contralateral long bone is even rarer (3). The present study describes a patient with DLBCL of the right femur, who achieved 7 years of complete remission following initial treatment with surgery and a standard chemotherapy regimen (six cycles, three weeks/cycle) consisting of cyclophosphamide $(1,200 \mathrm{mg}$, day 1$)$, doxorubicin ( $80 \mathrm{mg}$, day 1$)$, vincristine (2 $\mathrm{mg}$, day 1 ) and prednisolone (100 mg, days 3-7), plus the monoclonal anti-CD20 antibody rituximab (600 mg, day 0) (R-CHOP). However, the patient subsequently developed a new similar lesion in the left femur. No invasion of other bones or organs was observed throughout the disease course. To the best of our knowledge, this is the first report of this particular condition, and its underlying molecular mechanisms require additional study. Increasing knowledge regarding these mechanisms may provide novel 
strategies for the prevention of tumor metastasis and recurrence. Interventions based on these mechanisms may be able to decrease the growth and transfer of tumor cells, which may be of clinical significance.

\section{Case report}

A 65-year-old female presented to the Beijing Jishuitan Hospital (Beijing, China) in 2002 with a history of pain in the ventral side of the right thigh. Computed tomography (CT), emission CT (ECT), magnetic resonance imaging and positron emission tomography (PET) examinations all revealed an osteolytic lesion of the right upper femur and a pathological fracture of the femoral neck, without destruction of any other bones or organ involvement. The specimen from the femoral head, following femoral head replacement surgery, was hematoxylin and eosin stained using a horseradish catalase DAB Color kit (OriGene Technologies, Inc., Beijing, China). Uniform large cells were observed using a light microscope and immunohistochemistry revealed positive reactivity for leukocyte common antigen (LCA), cluster of differentiation (CD)20, CD79a and CD43, and no reactivity for $\mathrm{CD} 15, \mathrm{CD} 30$, vimentin, cytokeratin, $\mathrm{CD} 99$, S-100 protein or CD34. All primary and secondary antibodies (from the UltraSensitive ${ }^{\mathrm{TM}}$ S-P Hypersensitive Concentrated Kit: cat. no. KIT-0100M; dilution 1:1, ready-to-use; Goat anti Mouse IgG) were purchased from Fuzhou Maixin Biotech Co., Ltd., Fuzhou, China. The antibodies used were as follows: LCA (cat. no. Kit-0024; dilution 1:1,000), CD20 (cat. no. Kit-0001; dilution 1:300), CD79a (cat. no. MAB-0258; dilution 1:50), CD43 (cat. no. MAB-0032; dilution 1:200), CD15 (cat. no. MAB-0015; dilution 1:200), CD30 (cat. no.MAB-0023; dilution 1:4), vimentin (cat. no. Kit-0019; dilution 1:1), cytokeratin (cat. no. Kit-0009; dilution 1:1), CD99 (cat. no. MAB-0059; dilution 1:1), S-100 (cat. no. Kit-0007; dilution 1:1), CD34 (cat. no. Kit-0004; dilution 1:200). The primary antibodies were incubated for $60 \mathrm{~min}$ at room temperature or at $4^{\circ} \mathrm{C}$ overnight; secondary antibodies were incubated for $10 \mathrm{~min}$ at room temperature. The examination, which detected the expression of LCA, CD20, CD79a and CD43, enabled a definite diagnosis of primary DLBCL of the right femur (Fig. 1). Following the diagnosis, the patient received six cycles of CHOP (cyclophosphamide 1,200 mg, day 1; doxorubicin $80 \mathrm{mg}$, day 1; vincristine $2 \mathrm{mg}$, day 1; and prednisolone $100 \mathrm{mg}$, days 1-5) chemotherapy (three weeks/cycle), followed by eight cycles of rituximab (600 mg, day 0) (three weeks/cycle) as consolidation therapy. Subsequently, the patient achieved complete remission and was discharged from hospital.

In June 2009, 7 years after the initial diagnosis, the patient presented to the Union Hospital, Tongji Medical College, Huazhong University of Science and Technology (Wuhan, China) with pain in the left femur, similar to the symptoms experienced in the right femur 7 years prior. Routine examinations and peripheral blood and bone marrow cytology smears all appeared normal. B-mode ultrasonography of the superficial lymph nodes revealed several swollen lymph nodes located in the left side of the neck and the right groin. ECT of the bone demonstrated there was a focal enhancement in the upper left leg. A PET-CT scan revealed an area of bone destruction in the left femoral head, femoral neck and the upper femur, with a region of abnormal density in the bone marrow cavity. In addition, the PET-CT scan revealed multiple bilateral femoral lymph nodes with increased metabolic activity. As was observed for the previous lesion 7 years prior, there was no apparent destruction of additional bones or organs. Considering the patient's medical history and the existing test results, the patient was diagnosed with PLB of the left femur. It was hypothesized that the previous PLB of the right femur had recurred and transferred to the left femur (Fig. 2).

Subsequently, eight 6-day cycles of standard rituximab (600 mg per cycle)-CHOP chemotherapy were administered to the patient. $\mathrm{R}$ stands for the rituximab, and this regime can increase the efficiency of the chemotherapy using a CHOP.

However, in this regimen, liposomal adriamycin was used instead of doxorubivin in order to reduce cardiotoxicity, although there were no corresponding symptoms. The dosage of the liposomal adriamycin was $40 \mathrm{mg} / \mathrm{cycle}$ and the doses of other medicines are same as the aforementioned CHOP regime. Following chemotherapy, the patient improved; although PET-CT performed on 8th September, 2009 and 10th December, 2009 continued to demonstrate bone destruction in the left femoral head, femoral neck and upper femur with abnormal density in the bone marrow cavity, the abnormal metabolic activity was decreased compared with the scan prior to treatment. A subsequent PET-CT scan, performed on 5th May, 2010, revealed that the patient's condition had further improved. On the basis of chemotherapy, radiation therapy (30 Gy/10 f) was used intermittently. Following radiation therapy, rituximab was used exclusively as consolidation therapy for a total of 6 cycles (600 mg each cycle, three months apart). Though the patient had not achieved complete remission, the patient's general condition improved and no other sites of recurrence were identified in the subsequent follow-up $\sim 7$ years after treatment finished. The second femoral head replacement surgery was refused by the patient. Written informed consent was obtained from the patient for publication of the present study.

\section{Discussion}

PLB is a rare type of malignant bone tumor and most commonly occurs in patients aged $\sim 55$ years. PLB typically presents with localized ostealgia and primarily invades the bone, with or without cortical invasion or soft tissue extension. Regional lymph nodes or remote organs are not typically affected $(2,8,9)$. In addition to pathological fractures, patients with PLB may present with systemic symptoms, including fever, emaciation and night sweats (10). X-ray examination typically reveals widespread osteolytic lesions located in the axial skeleton or the metaphysis of long bones (11). However, due to the low morbidity, local symptoms, including pain and difficulty of movement at the lesion location, and inconspicuous systemic symptoms, PLB is difficult to specifically diagnose using radiography, and the final diagnosis usually depends on the histopathological assessment of biopsy specimens (5).

The patient in the present study was an elderly female with PLB. The lesion was initially identified in the right femur, and the patient achieved a complete remission following femoral head replacement and systemic treatment, including chemotherapy and targeted rituximab therapy. However, similar symptoms developed in the same portion of the left femur after $\sim 7$ years of complete remission. The patient was diagnosed with PLB on the basis of the symptoms and related clinical findings. PLBs in the 


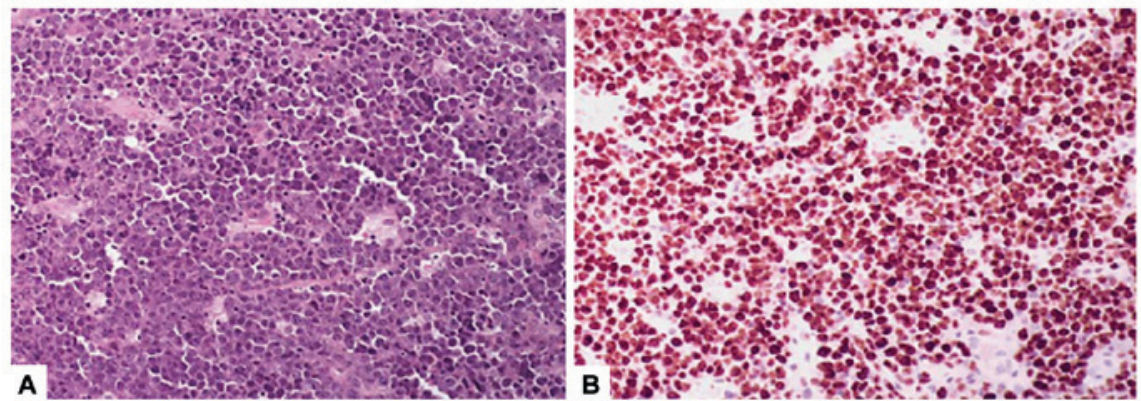

Figure 1. Pathological staining of a specimen from the femoral head of a patient with primary non-Hodgkin lymphoma of the bone. (A) Hematoxylin and eosin staining of a pathological section of the femoral head. This figure show uniform large cells, clear nucleoli and nuclear debris. (B) Ki-67 staining of the tumor cells. The ratio of tumor cells that are stained brown to those that are not is the Ki-67 index. Magnification, $\mathrm{x} 40$.

A

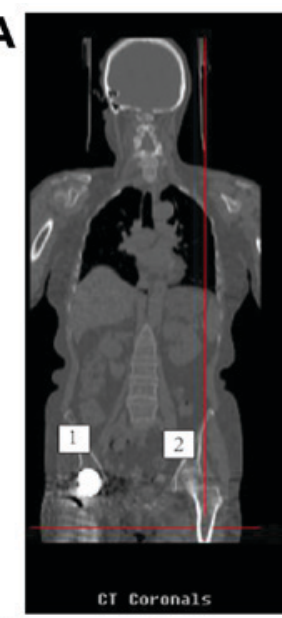

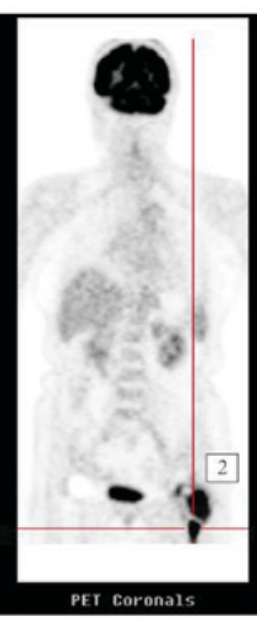

C

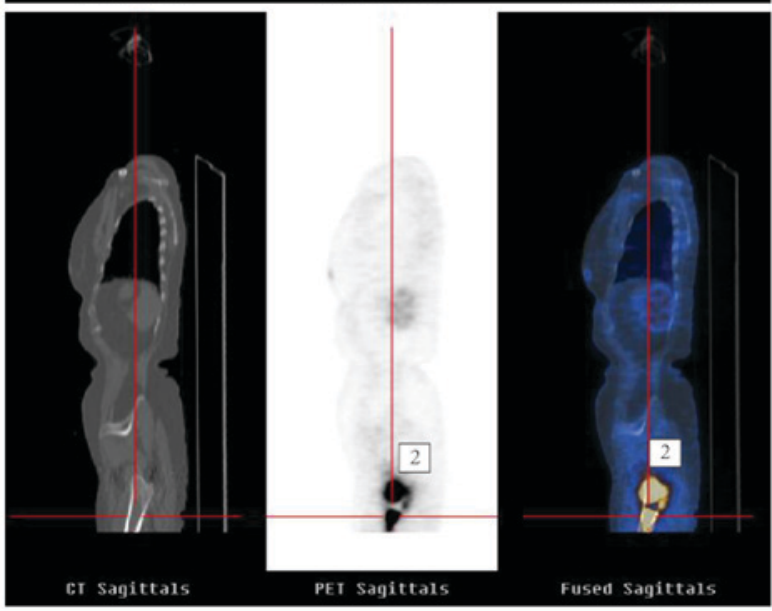

B

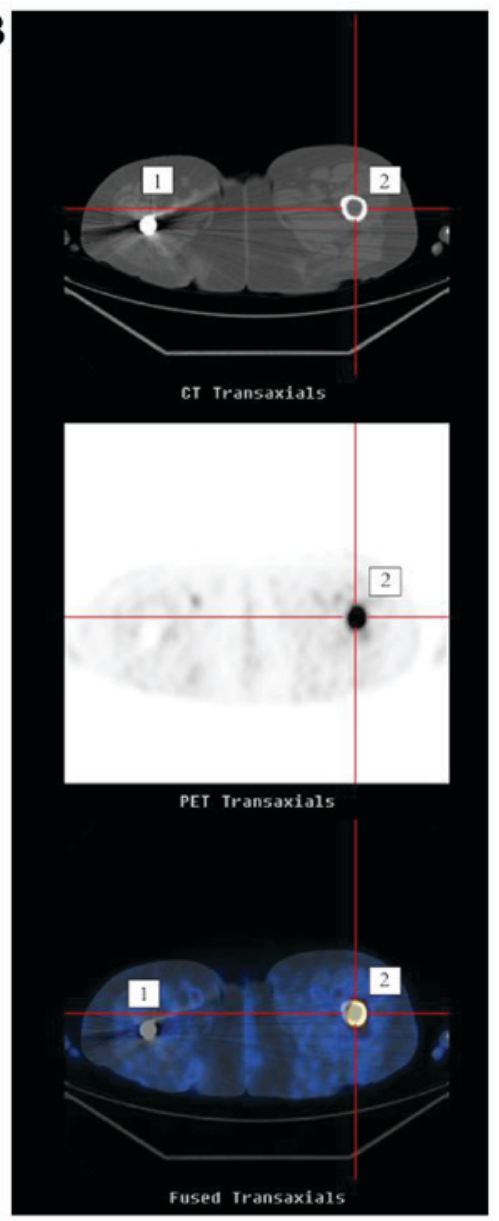

Figure 2. Positron emission tomography-computed tomography examination of a patient with primary non-Hodgkin lymphoma of the bone. In August 2009, a segment of bone destruction was identified on the left femoral head, femoral neck and upper femur with an area of abnormal density in the bone marrow cavity. In addition, multiple bilateral femoral lymph nodes with abnormally increased metabolic activity were identified. In the images, ' 1 ' indicates the femoral head replaced after the right femoral PLB, and ' 2 ' indicates the new lesion identified in the left femur. (A) Coronal, (B) transaxial and (C) sagittal images are shown.

spine, hip, ribs and skull have previously been identified (12); however, to the best of our knowledge, metastasis of the primary focus to the contralateral side has not been described. Therefore, the present case is extremely rare. It was initially unclear whether the second PLB lesion in the patient was a new lesion or metastasis. However, as the second lesion was of the same pathological type and in a similar region of the body to the first instance of PLB, and exhibited a successful response to the R-CHOP chemotherapy regimen, it was considered that the second case of PLB was due to a contralateral transfer according to the monophyletic theory.

Previous studies have suggested that circulating tumor cells (CTCs) tend to migrate back to the primary tumor site, or to an area similar to the original tumor microenvironment, by various mechanisms, including lymphocyte homing and self-seeding; such mechanisms depend on the chemokines, adhesion molecules and macrophages that exist in the microenvironment. The aforementioned mechanisms can lead to the recurrence and metastasis 
of the tumor. In addition, mesenchymal stem cells (MSCs) may serve a function in the progression of malignancy (13-19). The present patient had received treatment consisting of femoral head replacement and chemotherapy following the initial diagnosis. Thus, the original microenvironment in the patient's right femur was destroyed, and was not suitable for CTC engraftment. As a consequence, the cancer cells migrated to a similar microenvironment to that of the original PLB; following the establishment of CTCs in the left femur, the rich blood supply and appropriate microenvironment enabled the tumor cells to survive and proliferate, resulting in a new lesion in the left femur of the patient. The patient therefore experienced cancer relapse, and received 8 cycles of CHOP plus rituximab, in addition to radiation therapy, resulting in remission.

Previous studies have demonstrated that tumor microvascular endothelial cells have an increased permeability compared with any other location in primary malignant tumors $(20,21)$. These characteristics may enable the transfer of primary tumor cells through the endothelium and into the blood to become CTCs, and also cause the CTCs to gravitate to locations that are similar to the tumor site, or to the original tumor itself (22-24). Lymphocyte homing and self-seeding also serve notable roles in the metastasis and recurrence of malignancies (25). These two mechanisms may facilitate the transfer of CTCs to the original tumor site or to similar sites, and lymphatic-endothelial recognition serves a key function during this process $(13,15,16,26)$. Furthermore, a previous study revealed that chemokines and adhesion molecules, which are components of the tumor microenvironment, contribute to the migration and homing of malignant B lymphocytes (19); these molecules include L-selectin, C-X-C chemokine receptor type 4 , lymphocyte function-associated antigen $1, \mathrm{C}-\mathrm{C}$ chemokine receptor type (CCR)7 and CCR2 (19). Additionally, the microenvironment is crucial for the survival of malignant $\mathrm{T}$ and B lymphocytes (27). In recent years, the interaction between the tumor microenvironment and MSCs, and the functions in tumor growth and progression, have been identified (18). Tumor necrosis factor (TNF)- $\alpha$ in the tumor microenvironment may cause MSCs in healthy tissues to acquire similar functions to those of MSCs in the tumor stroma, and thereby accelerate the growth and progression of the neoplasm (18). The co-existence and interactions between the aforementioned mechanisms may explain how the present patient developed recurrence of PLB in a similar anatomical region following a period of complete remission for 7 years.

The study of the aforementioned mechanisms may provide novel strategies for preventing tumor metastasis and recurrence. Previous studies have demonstrated that the interaction between cancer cells and the stromal cells of the tumor microenvironment is required for the survival and localization of cancer cells (27-29). Recently, targeted treatments based on cytokines and adhesion molecules have been investigated, leading to improvements in the treatment of tumors and inhibition of tumor metastasis (30). Donate et al identified that junctional adhesion molecule (JAM)-C, which is produced by the vascular endothelium and by human B lymphocytes, is required for B lymphocyte homing. Furthermore, to some extent, the use of anti-JAM-C antibodies could restrict B cell migration and adhesion to the bone marrow, lymphoid organs and spleen (30). Cilengitide, an anti-angiogenic inhibitor of integrins $\alpha v \beta 3$ and $\alpha v \beta 5$, has also been used clinically (31). Additionally, thalidomide, which is used to treat multiple myeloma, following rituximab treatment has been identified to exhibit an anticancer effect in lymphoma via interfering with the microenvironment, inhibiting TNF- $\alpha$ and decreasing the induction of adhesion molecules (32). Furthermore, owing to the high permeability of the tumor vasculature, anti-angiogenic therapy can reduce CTCs to engraft and the original tumor to transfer (33). The tumor microenvironment and tumor angiogenesis have been demonstrated to serve significant roles in a number of types of solid tumor, including prostate, breast and ovarian cancers (34-37).

In summary, the current study presented a case of PLB that metastasized from one femur to the contralateral femur after 7 years of complete remission, which is an extremely rare manifestation. Various studies have researched the interplay between the tumor microenvironment and lymphocyte homing, self-seeding and MSCs, and have demonstrated that these mechanisms can contribute to the growth, progression, recurrence and metastasis of tumors. The previous studies may provide an explanation for the occurrence of metastasis in the present case. In recent years, there has been increasing interest in treatments based on these mechanisms. Exploitation of the underlying molecular mechanisms that enable the migration and engraftment of cancer cells may facilitate the development of novel anticancer strategies (such as interference with the tumor microenvironment or the production of antibodies against key cell surface molecules) that could be used to reduce the recurrence and metastasis of the disease.

\section{Acknowledgements}

The present case study was supported by the National Natural Sciences Foundation of China (grant no. 81000211).

\section{References}

1. Aziz F: Primary non-Hodgkin lymphoma of the bone. J Cancer Res Ther 5: 331, 2009.

2. Bruno Ventre M, Ferreri AJ, Gospodarowicz M, Govi S, Messina C, Porter D, Radford J, Heo DS, Park Y, Martinelli G, et al: Clinical features, management, and prognosis of an international series of 161 patients with limited-stage diffuse large B-Cell lymphoma of the bone (the IELSG-14 study). Oncologist 19: 291-298, 2014.

3. Hwang J, Cho S, Kim O, Shim HJ, Lee SR, Ahn JS, Yang DH, Kim YK, Lee JJ, Kim HJ and Chung IJ: Newly developed multiple myeloma in a patient with primary T-Cell lymphoma of bone. J Korean Med Sci 23: 544-547, 2008.

4. Fletcher CD: The evolving classification of soft tissue tumours: An update based on the new WHO classification. Histopathology 48: 3-12, 2006.

5. Stein ME, Kuten A, Gez E, Rosenblatt KE, Drumea K, Ben-Shachar M, Zidan J, Haim N and Epelbaum R: Primary lymphoma of bone: A retrospective study. Oncology 64: 322-327, 2003.

6. Beal K, Allen L and Yahalom J: Primary bone lymphoma: Treatment results and prognostic factors with long-term follow-up of 82 patients. Cancer 106: 2652-2656, 2006.

7. Wu H, Zhang L, Shao H, Sokol L, Sotomayor E, Letson D and Bui MM: Prognostic significance of soft tissue extension, international prognostic index, and multifocality in primary bone lymphoma: A single institutional experience. Br J Haematol 166: 60-68, 2014.

8. Vertinksy AT, Krasnokutsky MV, Augustin M and Bammer R: Cutting-edge imaging of the spine. Neuroimaging Clin N Am 17: 117-136, 2007.

9. Jawad MU, Schneiderbauer MM, Min ES, Cheung MC, Koniaris LG and Scully SP: Primary lymphoma of bone in adult patients. Cancer 116: 871-879, 2010. 
10. Zhang X, Zhu J, Song Y, Ping L and Zheng W: Clinical characterization and outcome of primary bone lymphoma: A retrospective study of 61 Chinese patients. Sci Rep 6: 28834, 2016.

11. Zinzani PL, Carrillo G, Ascani S, Barbieri E, Tani M, Paulli M, Stefoni V, Sabattini E, Alinari L, Binazzi R, et al: Primary bone lymphoma: Experience with 52 patients. Haematologica 88: 280-285, 2003

12. Baar J, Burkes RL, Bell R, Blackstein ME, Fernandes B and Langer F: Primary non-Hodgkin lymphoma of the bone. A clinicopathologic study. Cancer 73: 1194-1199, 1994.

13. Salmi $\mathrm{M}$ and Jalkanen S: Lymphocyte homing to the gut: Attraction, adhesion, and commitment. Immunol Rev 206 100-113, 2005

14. Piovan E, Tosello V, Indraccolo S, Masiero M, Persano L, Esposito G, Zamarchi R, Ponzoni M, Chieco-Bianchi L, Dalla-Favera $\mathrm{R}$ and Amadori A: Differential regulation of hypoxia-induced CXCR4 triggering during B-Cell development and lymphomagenesis. Cancer Res 67: 8605-8614, 2007.

15. Norton L and Massagué J: Is cancer a disease of self-seeding? Nat Med 12: 875-878, 2006.

16. Leung CT and Brugge JS: Tumor self-seeding: Bidirectional flow of tumor cells. Cell 139: 1226-1228, 2009.

17. Carmellet P and Jain RK: Angiogenesis in cancer and other diseases. Nature 407: 249-257, 2000.

18. Ren G, Zhao X, Wang Y, Zhang X, Chen X, Xu C, Yuan ZR, Roberts AI, Zhang L, Zheng B, et al: CCR2-dependent recruitment of macrophages by tumor-educated mesenchymal stromal cells promotes tumor development and is mimicked by TNF $\alpha$. Cell Stem Cell 11: 812-824, 2012.

19. Burger JA and Gribben JG: The microenvironment in chronic lymphocytic leukemia (CLL) and other B cell malignancies: Insight into disease biology and new targeted therapies. Semin Cancer Biol 24: 71-81, 2014.

20. Reddy BY, Lim PK, Silverio K, Patel SA, Won BW and Rameshwar P: The microenvironmental effect in the progression, metastasis, and dormancy of breast cancer: A model system within bone marrow. Int J Breast Cancer 2012: 721659, 2012.

21. Bates DO, Lodwick D and Williams B: Vascular endothelial growth factor and microvascular permeability. Microcirculation 6: 83-96, 1999.

22. Blagoev KB: Organ aging and susceptibility to cancer may be related to the geometry of the stem cell niche. Proc Natl Acad Sci USA 108: 19216-19221, 2011.

23. Joosse SA and Pantel K: Biologic challenges in the detection of circulating tumor cells. Cancer Res 73: 8-11, 2013.

24. van Laarhoven HW, Gambarota G, Heerschap A, Lok J, Verhagen I, Corti A, Toma S, Gallo Stampino C, van der Kogel A and Punt CJ: Effects of the tumor vasculature targeting agent NGR-TNF on the tumor microenvironment in murine lymphomas. Invest New Drugs 24: 27-36, 2006.
25. Dondossola E, Dobroff AS, Marchiò S, Cardó-Vila M, Hosoya H, Libutti SK, Corti A, Sidman RL, Arap W and Pasqualini R: Self-targeting of TNF-releasing cancer cells in preclinical models of primary and metastatic tumors. Proc Natl Acad Sci USA 113: 2223-2228, 2016

26. Kim MY, Oskarsson T, Acharyya S, Nguyen DX, Zhang XH, Norton L and Massagué J: Tumor self-seeding by circulating cancer cells. Cell 139: 1315-1326, 2009.

27. Blonska M, Agarwal NK and Vega F: Shaping of the tumor microenvironment: Stormal cells and vessels. Semin Cancer Biol 34: 3-13, 2015.

28. Mao Y, Keller ET, Garfield DH, Shen K and Wang J: Stromal cells in tumor microenvironment and breast cancer. Cancer Metastasis Rev 32: 303-315, 2013.

29. Kamińska K, Szczylik C, Bielecka ZF, Bartnik E, Porta C, Lian F and Czarnecka AM: The role of the cell-cell interactions in cancer progression. J Cell Mol Med 19: 283-296, 2015.

30. Doñate C, Ody C, Mckee T, Ruault-Jungblut S, Fischer N, Ropraz P, Imhof BA and Matthes T: Homing of human B cells to lymphoid organs and B-cell lymphoma engraftment are controlled by cell adhesion molecule JAM-C. Cancer Res 73: 640-651, 2013.

31. Desgrosellier JS and Cheresh DA: Integrins in cancer: Biological implications and therapeutic opportunities. Nat Rev Cancer 10: 9-22, 2010.

32. Kaufmann H, Raderer M, Wöhrer S, Püspök A, Bankier A, Zielinski C, Chott A and Drach J: Antitumor activity of rituximab plus thalidomide in patients with relapsed/refractory mantle cell lymphoma. Blood 104: 2269-2271, 2004.

33. Coupland SE: The challenge of the microenvironment in B-cell lymphomas. Histopathology 58: 69-80, 2011.

34. Efstathiou E, Troncoso P, Wen S, Do KA, Pettaway CA, Pisters LL, McDonnell TJ and Logothetis CJ: Initial modulation of the tumor microenvironment accounts for thalidomide activity in prostate cancer. Clin Cancer Res 13: 1224-1231, 2007.

35. Funahashi Y, Okamoto K, Adachi Y, Semba T, Uesugi M, Ozawa Y, Tohyama O, Uehara T, Kimura T, Watanabe H, et al: Eribulin mesylate reduces tumor microenvironment abnormality by vascular remodeling in preclinical human breast cancer models. Cancer Sci 105: 1334-1342, 2014.

36. Zhu Y, Li F, Vadakkan TJ, Zhang M, Landua J, Wei W, Ma J, Dickinson ME, Rosen JM and Lewis MT: Three-dimensional vasculature reconstruction of tumour microenvironment via local clustering and classification. Interface Focus 3: 20130015 , 2013.

37. Gavalas NG, Liontos M, Trachana SP, Bagratuni T, Arapinis C, Liacos C, Dimopoulos MA and Bamias A: Angiogenesis-related pathways in the pathogenesis of ovarian cancer. Int J Mol Sci 14: 15885-15909, 2013. 